\title{
A clinical trial comparing complete revascularization at the time of primary percutaneous coronary intervention versus during the index hospital admission in patients with multi-vessel coronary artery disease and ST-elevation myocardial infarction uncomplicated by cardiogenic shock
}

\author{
Mihnea Traian Nichita-Brendea1 (iD, Mircea I. Popescu1, 2 (D), Virgil Popa1 (D), \\ Polojintef Corbu Dorina Carmen ${ }^{1,2}$ (1)
}

${ }^{1}$ Department of Cardiology, Emergency Clinical County Hospital; Oradea-Romania

${ }^{2}$ Department of Medical Disciplines, Faculty of Medicine and Pharmacology, University of Oradea; Oradea-Romania

\section{ABSTRACT}

Objective: In this study, we aimed to compare major adverse cardiac and cerebrovascular events (MACCE), defined as a composite of death, stroke, myocardial infarction and symptom-induced revascularization, and mortality within one year of randomization between two strategies; complete revascularization including non-culprit lesions percutaneous coronary intervention (PCI) during primary PCI (PPCI) versus complete revascularization during the same hospital admission in patients with multi-vascular coronary artery disease (MVD) presenting with ST-elevation myocardial infarction (STEMI) uncomplicated by cardiogenic shock.

Methods: We randomized in a 1:1 manner 100 patients with MVD and STEMI uncomplicated by cardiogenic shock who had undergone successful culprit-lesion $\mathrm{PCl}$ to either a strategy of complete revascularization with $\mathrm{PCl}$ of angiographically significant non-culprit lesions in the index $\mathrm{PPCl}$ procedure or to a strategy of complete revascularization during a second procedure that took place during the same hospital admission. Results: The first primary outcome was death within a timeframe of one year and the second a composite of MACCE within a year following complete revascularization. Of the total number of patients monitored, $4 \%$ in each of the two groups was associated with the first primary outcome ( $p=0.984)$ and the second primary outcome in $6 \%(p=0.970)$. There was no statistical difference between outcomes in the two groups. Conclusion: Among patients with MVD and STEMI uncomplicated by cardiogenic shock, there was no difference regarding outcomes when using a strategy of complete revascularization of non-culprit lesions during PPCI or the same hospital admission.

Keywords: primary percutaneous coronary intervention, multi-vessel coronary artery disease, ST-elevation myocardial infarction

Cite this article as: Nichita-Brendea MT, Popescu MI, Popa V, Carmen PCD. A clinical trial comparing complete revascularization at the time of primary percutaneous coronary intervention versus during the index hospital admission in patients with multi-vessel coronary artery disease and ST-elevation myocardial infarction uncomplicated by cardiogenic shock. Anatol J Cardiol 2021; 25: 781-8.

\section{Introduction}

Patients presenting with ST-elevation myocardial infarction (STEMI) are effectively treated with emergency angioplasty, which restores blood flow to the coronary artery and in turn rescues the myocardium supplied by the infarct-related artery (IRA). One of the most important landmarks in the treatment of patients with acute STEMI was the use of primary percutaneous coronary intervention (PPCI), which showed an improvement in outcome compared with pharmacological reperfusion, "provided it could be performed expeditiously by an experienced team" (1). Among patients presenting with acute STEMIs, $20 \%$ to $50 \%$ have multi-vessel coronary artery disease (MVD) $(2,3)$. Several studies, including “The Preventive

Address for Correspondence: Mihnea Traian Nichita Brendea, MD, 


\section{HIGHLIGHTS}

- Complete revascularization has been shown to improve outcomes in ST-elevation myocardial infarction (STEMI) without shock.

- The optimal timing of treatment for non-culprit lesions is not known.

- There was identical one-year major adverse cardiac and cerebrovascular events in STEMI regardless of the timing of the complete revascularization strategy.

- There was identical one-year death in STEMI regardless of the timing of the complete revascularization strategy.

Angioplasty in Acute Myocardial Infarction (PRAMI)," (4) "The complete revascularization versus treatment of the culprit lesion only in patients with ST-segment elevation myocardial infarction and multi-vessel disease (DANAMI-3-PRIMULTI)," (5) "The Complete Versus Lesion-Only Primary PCI Trial (CvLPRIT)," (6) "The Comparison Between FFR Guided Revascularization Versus Conventional Strategy in Patients with Acute STEMI and Multi-vessel Disease (COMPAREACUTE)," (7) "Complete Versus Culprit-Only Revascularization Strategies to Treat Multi-vessel Disease after Early PCI for STEMI (COMPLETE)," (8) among many others have shown the benefits in terms of a lower rate of major adverse cardiac and cerebrovascular events (MACCE) in patients with a complete revascularization strategy versus an IRA only $\mathrm{PCl}$ strategy, thus causing a shift in the guidelines toward a strategy of complete revascularization in patients with MVD. To our knowledge, there was no head-to-head comparison of outcomes between different types of strategies for attaining complete revascularization in these patients; some studies completed the revascularization during the PPCI (PRAMI and COMPAREACUTE) and others in subsequent sessions (DANAMI-3PRIMULTI and CVLPRIT). However, there is a gap in the evidence body about the optimal time when non-IRA lesions [evaluated either by angiography or FFR according to the 2020 STEMI European Society of Cardiology (ESC) guidelines] should be revascularized (either immediately during the index $\mathrm{PCI}$ or staged at a later time per the STEMI ESC 2020 guidelines). If we apply a staged procedure strategy, we do not know the time interval from the PPCI when we should complete the revascularization process or if we should do it in one or more procedures.

In this study, we aimed to compare the occurrence of MACCE, defined as a composite of death, stroke, myocardial infarction and symptom induced revascularization, and the incidence of mortality within a one-year time limit from the PPCI procedure among patients with MVD and STEMI uncomplicated by shock, who had undergone successful complete revascularization of all angiographically significant lesions during the index PPCI (group A) and the ones who had benefited from a staged
$\mathrm{PCl}$ strategy - treatment of the culprit vessel during PPCl and non-culprit lesions in a second procedure, done before hospital discharge (group B).

\section{Methods}

\section{Trial design}

Our study was a single-center, prospective, randomized in a 1:1 fashion trial that compared complete revascularization of all (including non-culprit) stenosis during the index $\mathrm{PPCl}$ with complete revascularization in a second $\mathrm{PCl}$ session that took place during the same hospital admission as the PPCl in patients with MVD and STEMI uncomplicated by cardiogenic shock who had undergone successful culprit-lesion PPCI.

The protocol received approval from the hospital's Ethics Committee and was registered in a clinical trials database. The authors of this paper contributed to the study's design and were also responsible for gathering, analyzing, and preparing the manuscript to be submitted for publication. The authors take full responsibility for the veracity of the data presented and the proper conduct by the trial's approved protocol.

Ethics approval and consent to participate: The trial's reference number is 20466/08.09.2020 and was approved by the Emergency Clinical County Hospital of Oradea's Ethics Committee. Written informed consent was obtained from study participants.

\section{Eligibility}

After successfully completing IRA PPCI, patients with MVD and STEMI uncomplicated by cardiogenic shock became eligible for being randomized in the trial. Multi-vessel coronary disease implied that a minimum of an angiographically significant non-culprit stenosis in a vessel larger than $2 \mathrm{~mm}$ in diameter, different from the IRA, was present and could be treated with $\mathrm{PCl}$. We appreciated as relevant the non-culprit lesions that caused more than $75 \%$ stenosis, by visual estimation on angiography [or $50 \%$ in the left main coronary artery (LMCA)], or stenosis ranging from $50 \%$ to $74 \%$ associated with a fractional flow reserve (FFR) evaluation below 0.8 . Inclusion and exclusion criteria are included in Appendix A. Written informed consent was obtained from all the study participants.

\section{Randomization and trial treatments}

Eligible patients were randomly assigned in a 1:1 fashion to either group $A$ receiving complete revascularization during the $\mathrm{PPCl}$ or group $\mathrm{B}$ undergoing complete revascularization in staged procedures (Fig. 1). In group B, complete revascularization was achieved in a single-stage procedure within 48-72 hours following PPCl. Our working algorithm was always to treat the IRA first; and after successful PPCl, the patients were randomized into the two groups.

Drug eluting stents (DESs) were preferred for all the $\mathrm{PCI}$ procedures, with bare-metal stents (BMSs) being used only in case of DES dimension unavailability. 


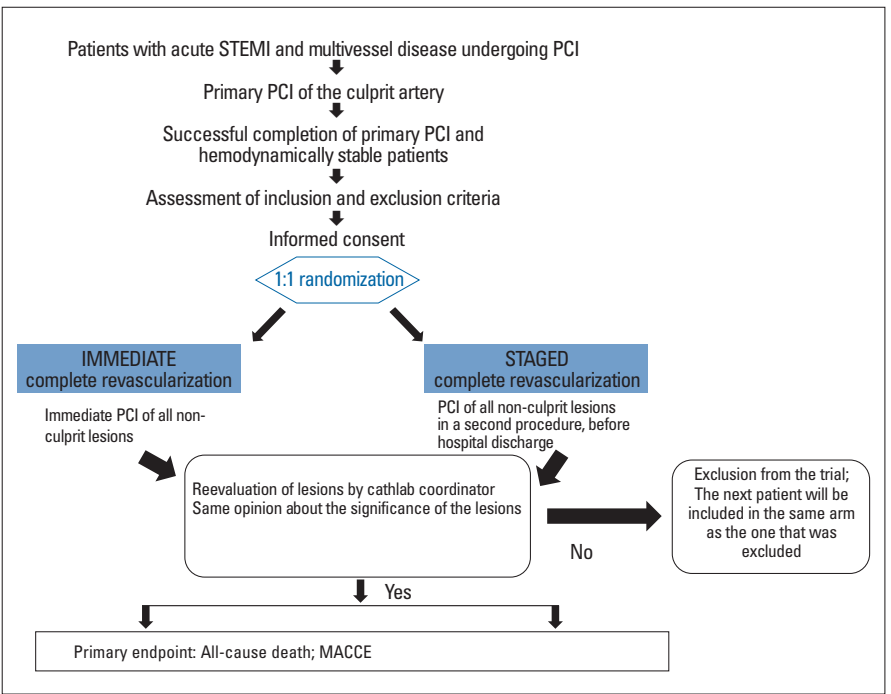

Figure 1. Study flow chart

MACCE - major adverse cardiac and cerebrovascular events; $\mathrm{PCl}$ - percutaneous coronary intervention; STEMI - ST-segment elevation myocardial infarction

$\mathrm{PCl}$ of chronic total occlusions (CTO) was attempted in all the patients with this type of lesion. The CTO lesions were approached at the operator's discretion, considering the hybrid algorithm, and were usually deferred as the last non-culprit lesions to be undertaken. We must specify that the operator was experienced in dealing with CTOs. The criteria for stopping the CTO procedure were if a contrast dose higher than $5 x$ body weight $(\mathrm{kg}) /$ serum creatinine $(\mathrm{mg} / \mathrm{dL})$ or an air kerma dose of 7 Gy had been reached without completion of the procedure, the occurrence of a procedural complication, or when the operator considered that further attempts were futile. All coronary angiographies were evaluated by a second operator (the catheterization laboratory coordinator) to assess the severity of coronary lesions and certifying which patients would remain in the trial for further follow-up (thus eliminating operator bias). Provisional stenting, TAP, Culotte, and DK-Crush techniques were used for bifurcation stenosis treatment, favoring provisional stenting whenever possible. All the procedures included simultaneous kissing balloon and final proximal optimization technique.

All the procedures were done by a single operator, thus eliminating operator bias. Hospital follow-up visits took place at 4 weeks and 1 year. Patients included in the trial received medical therapy in accordance with guideline recommendations.

\section{Outcomes}

The first primary outcome was all-cause death at one year, whereas the second primary outcome was a composite of allcause mortality, new myocardial infarction, stroke, and symptom-driven revascularization at one year. We defined myocardial infarction using the criteria from the fourth universal definition of myocardial infarction and further subdivided them according to the territory involved as it appeared on the ECG. One of the authors, a cardiac clinician who was not the operator and unaware of the treatment assignments, documented out- come events. We relied on rigorous clinical assessment and review of the medical files to evaluate outcomes at the onemonth and one-year follow-up visits. If one patient failed to participate in the follow-up visit, we interviewed via telephone and proceeded with the revision of the patient's medical files to ascertain whether an outcome event has occurred.

Although a safety endpoint was not included, there were no major bleedings, contrast-induced nephropathy, and access site complications reported. Contrast-induced nephropathy was defined as a rise of $25 \%$ in serum creatinine from the baseline value within 72 hours of the PCI. The attending physicians tracked the abovementioned safety outcomes and reported their presence to the author that oversaw the outcome monitoring.

\section{Statistical analysis}

Categorical data were reported as numbers (percentages) for its variables such as sex, death at one year, stroke, symptominduced revascularization, MACCE, myocardial infarction, number of vessels requiring $\mathrm{PCl}$, presence of one or more CTOs, left main disease, and involvement of one or more bifurcations. We used the Pearson chi-squared test for group comparison. Data for continuous variables were presented as mean \pm standard deviation when the distribution was uniform for age, number of DES used, and number of BMS used and as medians and interquartile range (IOR) when the distribution was not uniform for the corrected Syntax score. We performed a comparison of the central tendency of the baseline characteristics and endpoints of the two groups using the t-test for normally distributed continuous variables and the Mann-Whitney rank-sum test to compare the abnormally distributed continuous variables. We used the Kaplan-Meier method to assess the time to primary endpoints and create a survival estimate. The chi-squared test was used to compare outcomes in the two groups. A p-value $<0.05$ was considered statistically significant. The statistical analysis was done with MedCalc version 19.4.1 MedCalc Software LtdMedCalc ${ }^{\circledR}$ Statistical Software version 19.8 (MedCalc Software Ltd., Ostend, Belgium; https://www.medcalc.org; 2021).

\section{Results}

\section{Patients, treatment, and follow-up}

From January 01, 2017, to June 01, 2019, we enrolled 100 eligible patients from a single-center, the Clinical Emergency County Hospital, Oradea, who underwent randomization in a 1:1 manner in two groups; group A receiving complete revascularization during the $\mathrm{PPCl}$ and group $\mathrm{B}$ undergoing complete revascularization in staged procedures during the same admission as the PPCI.

After PPCl of the IRA, we calculated the Syntax and Society of Thoracic Surgeons (STS) score and provided the patient with information about the best course of action, considering that coronary artery bypass grafting (CABG) remains the gold standard of care for patients with three-vessel disease (9). Median Syntax score after PPCI for IRA (not taking into consideration lesions that were present on the IRA) was not significantly 
higher in group $A$ compared with group $B$ (10 vs. 9.487, $p=0.817$ ), from a statistical point of view. Successful CTO PCI was achieved in $80 \%$ of patients in group $A$ and $67 \%$ of patients in group B [ $95 \%$ confidence interval (Cl) $37.284 \%-61.876 \%$, $p=0.700$ ] There were 8 CTOs in 8 patients (none of the patients presented 2 or more CTOs) (4 CTOs were in the LAD and 4 in the RCA). Of these patients, 5 were in group A [2 LAD CTOs and 3 right coronary artery (RCA) CTOs] and 3 in group B (2 LAD CTOs and 1 RCA СТО). In both the groups, the PCI of an RCA CTO was not successful and was finished with subintimal plaque modification technique as an investment procedure.

There was no crossover between the two groups.

We monitored outcome endpoints up to the time of the last follow-up visit. There were no patients lost to follow up. Two patients provided medical records issued at one month and one year after hospital discharge by the primary healthcare provider (because their residence was outside the area where our facility provided healthcare).

\section{Baseline characteristics}

There were no significant statistical differences among baseline characteristics between the two groups of patients, except for a greater number of DESs used in group $B(4.14 \pm 1.8)$ versus group $A(3.3 \pm 1.5)(p=0.014)$ and left ventricular ejection fraction (LVEF) ( $p=0.031)$. Detailed baseline characteristics are presented in Table 1. The proportion of patients who got one or more BMSs implanted is $12 \%$, and one patient had only BMSs implanted (group B). There was no statistical difference among the two groups regarding the mean number of BMSs implanted (0.38 vs. $0.16, p=0.191)$. The reason behind the use of BMSs was a logistical one, because of the unavailability of a certain DES dimension (diameter) at the time of the procedure. When the available DES had the required diameter but not the necessary length, we preferred to implant 2 or more shorter DESs rather than a single BMS with the right length. This might partially explain the aforementioned difference between the number of DESs used. None of the LMCA, CTO, or bifurcation lesions were stented with BMSs.

No statistically significant differences were identified regarding the type (territory) of infarction, presence of CTO lesions, number of vessels involved (the number reflects the sum of the vessels including the IRA), bifurcation lesions, the involvement of the left main coronary artery (Fig. 2, 3, and 4) (Table 1).

The mean baseline LVEF (\%) was significantly higher for patients in group B than in those in group A $(42.040 ; 95 \% \mathrm{Cl}$ : 39.772-44.308 vs. $45.125 ; 95 \%$ Cl: 43.435-46.815) (Fig. 5).

We detected the first primary outcome in 2 patients of the total number of patients $(n=50)$ representing $4 \%$ of group $A$; whereas in group $B$ (50 patients), the event was also detected in 2 patients (4\%) (HR 0.979; 95\% Cl 0.13-6.95; $\mathrm{p}=0.983$ ) (Fig. 6). In both groups studied, we encountered the second primary outcome in 3 patients (accounting for $6 \%$ of each group) (hazard ratio $0.96 ; 95 \% \mathrm{Cl} 0.19-4.80 ; p=0.970$ ) (Fig. 7). There was no statistical difference between outcomes in the two groups.

\begin{tabular}{|lccc|}
\hline \multicolumn{4}{|l|}{ Table 1. Baseline characteristics } \\
\hline Characteristics & Group A & Group B & P-value \\
\hline $\begin{array}{l}\text { Number of cases, } \\
\mathrm{n}(\%)\end{array}$ & $50(50)$ & $50(50)$ & 1 \\
Sex (male) & & & \\
Medical history n (\%) & $37(74)$ & $36(72)$ & 0.823 \\
$\quad$ & $12(24)$ & $11(22)$ & 0.813 \\
$\quad$ Diabetes & $20(40)$ & $24(48)$ & 0.423 \\
Hypertension & $25(50)$ & $21(42)$ & 0.425 \\
$\quad$ Current smoker & & & \\
Type of STEMI n (\%) & $22(44)$ & $18(36)$ & 0.417 \\
$\quad$ Anterior & $22(44)$ & $30(60)$ & 0.111 \\
$\quad$ Inferior & $4(8)$ & $2(4)$ & 0.402 \\
$\quad$ Lateral & $2(4)$ & $0(0)$ & 0.155 \\
$\quad$ Posterior &
\end{tabular}

Number of diseased vessels, including IRA, $\mathrm{n}(\%)$

2

3

4

5

$\begin{array}{ccc}31(62) & 27(54) & 0.420 \\ 13(26) & 10(20) & 0.478 \\ 5(10) & 11(22) & 0.103 \\ 1(2) & 2(4) & 0.560 \\ 10(20) & 16(32) & 0.173\end{array}$

Bifurcation lesion

n $(\%)$

Number of DESs used

$3.3 \pm 1.5$

$4.14 \pm 1.8$

0.014

(mean \pm SD)

Number of BMSs used

0.38

0.16

0.191

(mean)

Total stent length

(median, IQR) (mm)

44.30

(36-144)

56.461

36-180)

Stent diameter

$3.2 \pm 0.9$

$3.1 \pm 0.5$

0.494

$($ mean $\pm S D)(m m)$

Syntax score after

PPCI (median, IQR)

Left ventricular

ejection fraction on

admission (\%)

Presence of CTOs, n (\%)

Left main PCl, n (\%)

10

9.487

0.817

(6.00-12.00)

(7.00-14.00)

$42.040 \quad 45.125$

0.031

(39.772-44.308) (43.435-46.815)

0.032

Successful CTO PCl

$5(10)$

$3(6)$

0.463

procedure $(\%)$

FFR-guided PCl, $n(\%)$

8 (16)

$10(20)$

0.604

80

67

0.700

BMS - bare-metal stent; CTO - chronic total occlusion; DES - drug-eluting stent; FFR fractional flow reserve; IRA - Infarct related artery; IQR- interquartile range; $n$ - number of patients; $\mathrm{PCl}$ - percutaneous coronary intervention; $\mathrm{PPCl}$ - primary percutaneous coronary intervention; SD - standard deviation; STEMI - ST-elevation myocardial infarction

We observed that survival at one year was identical in the two groups. The same observation can be made about the MACCE outcome. However, we observed a numerically higher rate of 


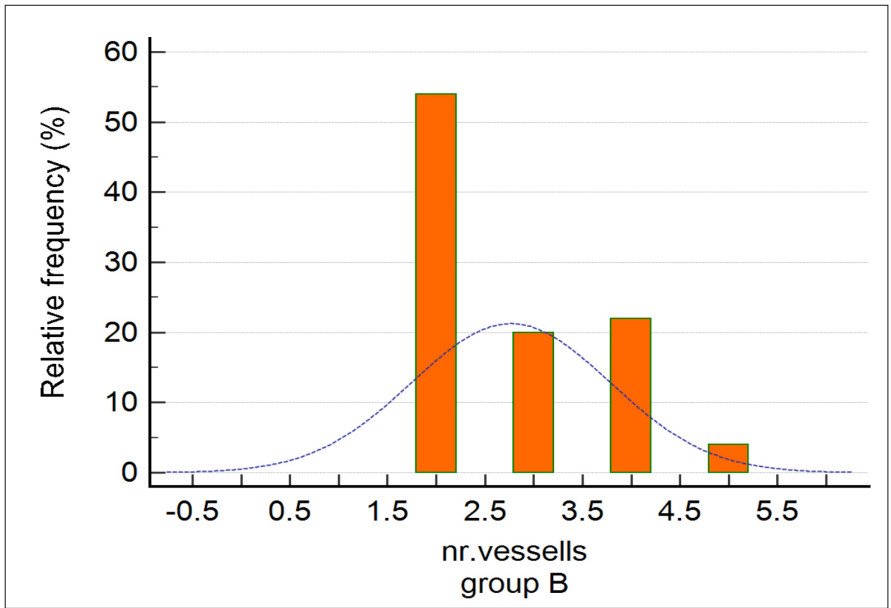

Figure 2. Relative frequencies of the number of vessels with significant angiographic stenosis in group B

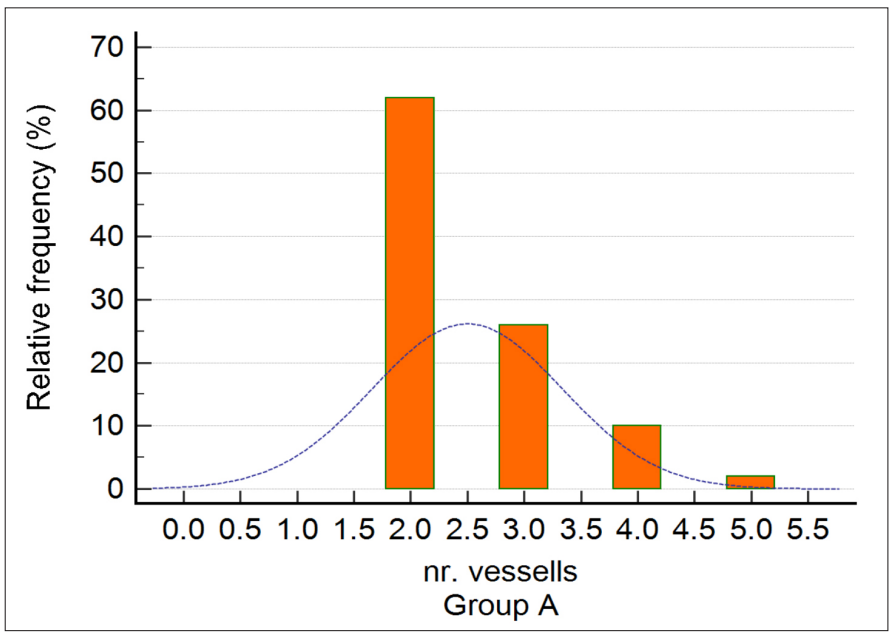

Figure 3. Relative frequencies of the number of vessels with significant angiographic stenosis in group $A$

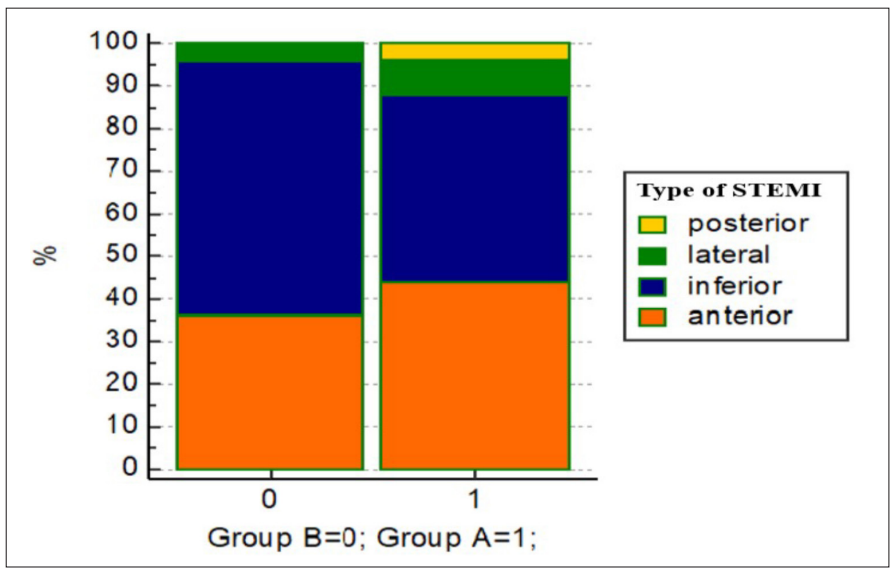

Figure 4. Comparison between the prevalence of infarction type in the two groups

stroke, although statistically not significant in group $A(p=0.332)$, whereas the incidence of symptom-driven revascularization was higher in group $B(p=0.307)$ also without statistical significance.

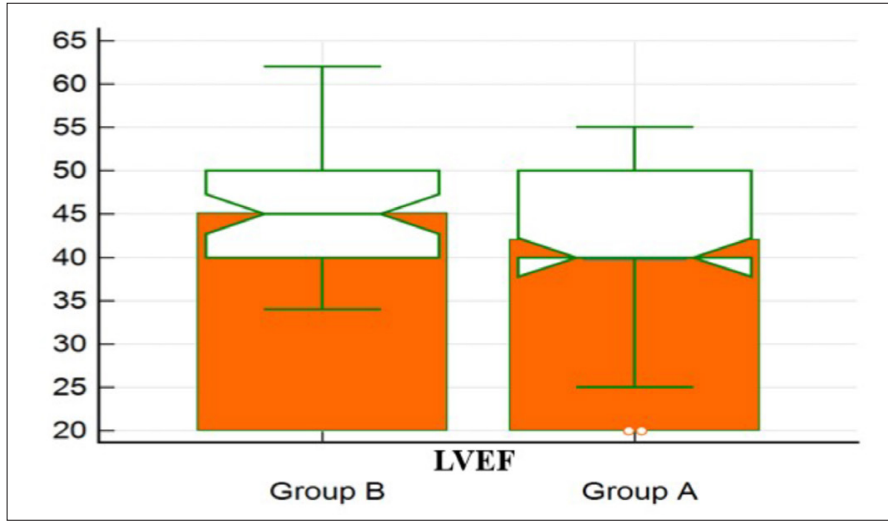

Figure 5. Comparison between the mean left ventricular ejection fraction in the two groups

LVEF - left ventricular ejection fraction

\section{Discussion}

Our trial showed that there was no statistical difference regarding the following endpoints; all-cause death at one year and a composite of all-cause mortality, stroke, myocardial infarction, and symptom-driven revascularization, at one year between the two groups. However, there was non-statistically significant better survival at one month with complete revascularization during $\mathrm{PPCl}$ strategy ( $\mathrm{p}=0.155$ ) (Fig. 6). This observation contrasted to the one made by Tarantini et al. (10) that a staged multi-vessel revascularization strategy may improve early and late survival. In the meta-analysis, 3 revascularization strategies were compared; 1) IRA-only $\mathrm{PCl}$, defined as $\mathrm{PCl}$ limited to the culprit lesion(s) only; 2) single procedure MV-PCl, defined as $\mathrm{PCI}$ in which lesions in the IRA as well as $\geq 1$ non-IRA vessels were treated during the STEMI index procedure; and 3) staged $\mathrm{MV}-\mathrm{PCl}$, defined as $\mathrm{PCl}$ limited to the IRA during the index procedure followed by planned $\mathrm{PCl}$ of significant non-IRA lesions at a different time; and Tarantini et al. (10) showed that the best results, both early and late, were achieved through the third revascularization strategy. Our trial showed no difference between strategy numbers 2 and 3 regarding late survival and better, although non-statistically significant, 30-day survival with strategy number 2 . We assume the difference comes from the difference in inclusion/exclusion criteria, study design (both prospective and retrospective trials were included in the metaanalysis), and so forth. On analyzing the different studies included in this meta-analysis, we identified one trial similar to ours, namely the one by Ochala et al. (11), "The function of the left ventricle after complete multi-vessel one-stage $\mathrm{PCl}$ in patients with acute myocardial infarction" which still differed in the fact that it did not include an all-comers MVD STEMI population (CTOs, long lesions were the exclusion criteria, and FFR was not used). This trial showed similar findings to ours, specifically that there was no difference between the 2 types of complete revascularizations investigated. Unfortunately, the trial in question was also underpowered, reflecting the difficulty in including a large number of patients in an interventional trial with a design that entails a single operator. All the other trials included in the 


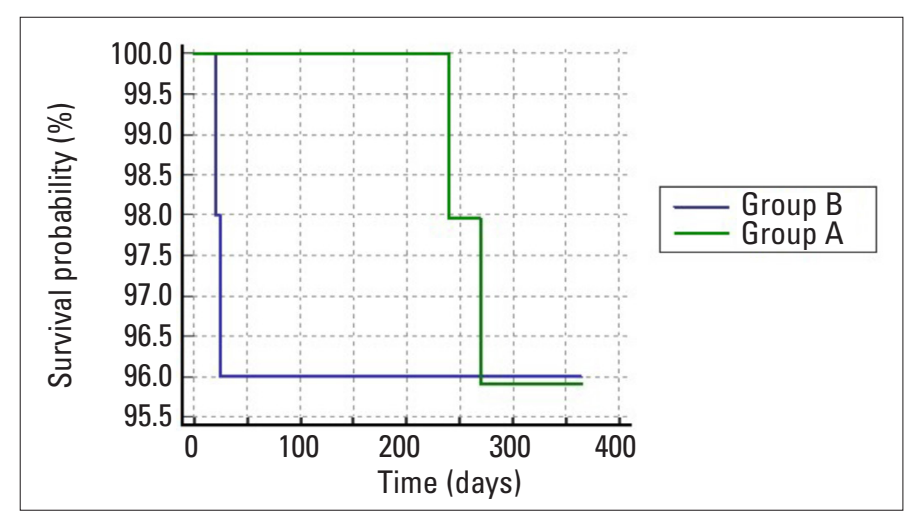

Figure 6. Kaplan-Meier curves comparing the primary outcome and survival at one year between the two groups of patients

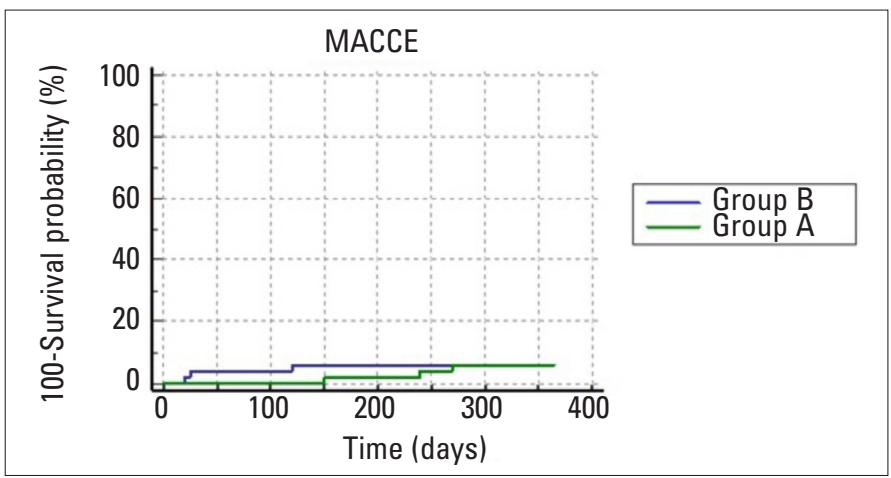

Figure 7. Kaplan-Meier curves comparing the second primary outcome and the incidence of major adverse cardiac and cerebrovascular events at one year between the two groups of patients

MACCE - major adverse cardiac and cerebrovascular events

meta-analysis had more than one operator performing the PCls.

We also observed a higher but statistically non-significant incidence of stroke in the complete revascularization during the $\mathrm{PPCl}$ group, whereas the staged PCI group presented a higher, albeit statistically non-significant, risk of symptom-driven revascularization. The higher incidence of stroke could be linked to a higher incidence of anterior wall infarction in group A. As the trial was underpowered, we could not make such an assumption; nevertheless, this association between anterior wall infarction and post-STEMI stroke was also reported by Hachet et al. (12).

The trial, although underpowered, concluded that among patients with MVD presenting with STEMI uncomplicated by cardiogenic shock, there was no difference between the strategy of complete revascularization used at the moment of the $\mathrm{PPCl}$ or staged during the same hospital admission.

The incidence of all-cause mortality at one year ( $4 \%$ vs. $5.1 \%$ vs. $1.3 \% ; p=0.666$ and $p=0.090$, respectively), MI ( $1 \%$ vs. $1.3 \%$ vs. $2.99 \% ; p=0.814$ and $p=0.277$, respectively) and symptom driven revascularization ( $1 \%$ vs. $5.12 \%$ vs. $4.7 \% ; p=0.076$ and $p=0.093$, respectively) was similar in our trial with those obtained in the complete revascularization arms of the PRAMI and CvLPRIT trials.

The fact that complete revascularization offers better outcomes has already been proven in a series of studies. However, the best way to achieve this completeness was not investigated
(4-8); however, it is not clear when non-IRA lesions should be revascularized; and if we use a staged procedure strategy according to the ESC guidelines, what is the time interval from the PPCl to the time of the complete revascularization process or if we should do one or more procedures. In this trial, we tried to fill that void in the evidence gap by showing similar results between an immediate complete revascularization strategy versus a staged approach in the index hospital admission among a non-selected population of patients with MVD presenting with STEMI uncomplicated by shock.

The undertaking of ad hoc CTO PCI (group A; in group B, there was a $48-72 \mathrm{hr}$ time frame for procedural set-up), especially in the setting of STEMI, is not indicated. However, we intended to compare the 2 strategies for attaining complete revascularization in an all-comers MVD patient population presenting with STEMI uncomplicated by cardiogenic shock. Thus, the presence of CTOs was not considered an exclusion criterion. This subset of lesions is frequently present in patients with MVD and STEMI. Therefore, we appreciated that by not including this particular high-risk category in our trial, we would have caused an important selection bias. Other trials, for example, COMPLETE, dealing with complete revascularization in the setting of STEMI, also included patients with CTOs. We did not assess procedural time or contrast media use; however, to limit the possible harmful effects, radiation dose was kept under $7 \mathrm{~Gy}$ for the whole procedure (CTO plus non-CTO lesions) and contrast media use under $5 \mathrm{x}$ body weight $(\mathrm{kg}) / \mathrm{serum}$ creatinine $(\mathrm{mg} /$ $\mathrm{dL})$. We also routinely employed prevention methods for reducing contrast-induced nephropathy (CIN), such as good hydration, statin, and ACC use. There were no reports from the treating physicians regarding $\mathrm{CIN}$ requiring renal replacement therapy, radiation-induced skin lesions, or bleeding complications in any of the patients (presenting with or without СТО). An interesting fact was that group $A$ (complete revascularization of all nonIRA significant stenoses during PPCI), in which ad hoc CTO-PCI was performed, had a higher success rate $(80 \%)$ versus group $B$ $(67 \%)$ (complete revascularization of non-IRA significant stenoses during a staged $\mathrm{PCI}$ procedure that took place during the index hospitalization). However, we subscribe to the idea that treating CTOs in the context of STEMI needs further investigation and is not advisable at this moment (results in terms of CTO $\mathrm{PCl}$ success from this trial have no statistical bearing and are owing to the small number of patients with this type of lesion analyzed in the study).

FFR measurement was employed in $11 \%$ of the patients. Following successful IRA PCl, non-culprit lesions were evaluated using quantitative coronary angiography (OCA). Eleven patients presented with stenoses that were deemed intermediate $(50 \%-74 \%$ by $\mathrm{QCA})$, and all of them were evaluated with an FFR measurement at the time of PPCI. Intracoronary administration of adenosine as a $200 \mu \mathrm{g}$ bolus was performed. We repeated the measurement 2 times for an accurate result. There were no problems encountered with the use of adenosine, except transitory AV block that did not require temporary pacemaker implantation. Of the 11 patients tested with FFR, 3 had a value $<0.8$ and 
subsequently underwent stenting. We employed functional assessment whenever a stenosis was considered intermediate on QCA, thus reducing overstenting or understenting. Unnecessary non-culprit lesion PCls can be reduced by an FFR guided strategy; however, it is safe to say that in this specific case, the influence of FFR based treatment decisions would have been minimal. Several studies in the literature have raised the question of whether FFR in the setting of STEMI produces reliable results. To our knowledge, most of them favor an FFR driven revascularization in this subset of patients (13-17).

Trials like DANAMI 3 PRIMULTI or COMPARE ACUTE also employed an FFR guided revascularization in the setting of STEMI.

\section{Study limitations}

The main limitation of this trial was the small number of patients investigated $(n=100)$. This is related to the fact that our center treats a mean number of 450 STEMIs/year. The workload is shared between 3 independent operators. Considering that only $20 \%-50 \%$ of patients have coronary multi-vessel disease and patients with cardiogenic shock or patients in which complete revascularization was considered unattainable or suited for CABG were not included in the trial, accounts for approximately 50 cases/year/operator. The patients included in the trial had the same operator to exclude operator bias as a result influencing factor. The trial duration, including the period of oneyear follow-up, took place over a period of 3 years.

In view of the fact that different operators have different skillsets and experience, all the procedures were done by a single operator to eliminate any operator bias. We tried to exclude operator bias as an influencing factor of the trial outcomes and determine if a particular strategy of obtaining complete revascularization is superior to the other. However, this approach entails several limitations regarding the external validity of our single-center study. The operator who performed the procedures included in this trial, as well as the center in which the study took place, fall in the category of high-volume operator/center (>100 PCl/year/operator and >200 PCl/ year/center) according to National Cardiovascular Data Registry. We estimate that similar results would be expected in different centers and by other operators falling in the same work volume category.

Our trial employed an FFR guided non-culprit PCI strategy in a relatively reduced number of patients $(11 \%)$. It is up for discussion if the physiological evaluation of non-culprit stenosis on a wider scale impacts the trial results.

\section{Conclusion}

This trial showed that there were no differences in MACCE or death at 1 year among patients with MVD and STEMI uncomplicated by cardiogenic shock who benefited from complete revascularization of all significant stenoses, either during the index $\mathrm{PPCl}$ or in staged procedures, with the non-IRA lesions being revascularized in a second procedure during the index hospitalization. Considering the results, we are encouraged to continue the study and include other operators to achieve ade- quate power for the trial. Albeit small, our trial could contribute to the idea that immediate complete revascularization is noninferior to staged complete revascularization in patients with MVD presenting with STEMI and uncomplicated by cardiogenic shock. The abovementioned hypothesis is investigated in 2 ongoing trials, "The Direct Complete versus Staged Complete Revascularization in Patients Presenting with Acute Coronary Syndromes and Multi-vessel Disease Trial (BioVasc)" and "The MULTivessel Immediate versus STAged RevaScularization in Acute Myocardial Infarction - The MULTISTARS AMI Trial (MULTISTARS AMI)," both of which bear similarities but are not identical to our study.

On the basis of our study's conclusions, we can state that the operator can safely choose the strategy for achieving complete revascularization considering different factors favoring deferral of non-culprit lesions for a second procedure like personal fatigue, presentation during the night shift, and need for better evaluation of coronary/lesion anatomy, which may require intracoronary imaging/plaque modification techniques that could be unavailable during the PPCl, operator expertise (nonCTO operator, junior staff operator), or favoring the treatment of all lesions during the index procedure like catheterization laboratory or operator availability, length of hospital stay, reimbursement, and the patient's medical insurance.

Conflict of interest: None declared.

Peer-review: Externally peer-reviewed.

Author contributions: Concept - M.T.N.B.; Design - M.T.N.B.; Supervision - V.P.; Fundings - None; Materials - None; Data collection \&/or processing - P.C.D.C.; Analysis \&/or interpretation - M.I.P., V.P., P.C.D.C.; Literature search - M.I.P., P.C.D.C.; Writing - M.T.N.B.; Critical review - M.I.P., P.C.D.C.

\section{References}

1. Ibanez B, James S, Agewall S, Antunes MJ, Bucciarelli-Ducci C, Bueno H, et al.; ESC Scientific Document Group. 2017 ESC Guidelines for the management of acute myocardial infarction in patients presenting with ST-segment elevation: The Task Force for the management of acute myocardial infarction in patients presenting with ST-segment elevation of the European Society of Cardiology (ESC). Eur Heart J 2018; 39: 119-77. [Crossref]

2. Martí D, Mestre JL, Salido L, Esteban MJ, Casas E, Pey J, et al. Incidence, angiographic features and outcomes of patients presenting with subtle ST-elevation myocardial infarction. Am Heart J 2014; 168: 884-90. [Crossref]

3. Park DW, Clare RM, Schulte PJ, Pieper KS, Shaw LK, Califf RM, et al. Extent, location, and clinical significance of non-infarct-related coronary artery disease among patients with ST-elevation myocardial infarction. JAMA 2014; 312: 2019-27. [Crossref]

4. Wald DS, Morris JK, Wald NJ, Chase AJ, Edwards RJ, Hughes LO, et al.; PRAMI Investigators. Randomized trial of preventive angioplasty in myocardial infarction. N Engl J Med 2013; 369: 1115-23. [Crossref]

5. Engstrøm T, Kelbæk H, Helqvist S, Høfsten DE, Kløvgaard L, Holmvang L, et al.; DANAMI-3-PRIMULTI Investigators. Complete revascularisation versus treatment of the culprit lesion only in 
patients with ST-segment elevation myocardial infarction and multivessel disease (DANAMI-3-PRIMULTI): an open-label, randomised controlled trial. Lancet 2015; 386: 665-71. [Crossref]

6. Gershlick AH, Khan JN, Kelly DJ, Greenwood JP, Sasikaran T, Curzen N, et al. Randomized trial of complete versus lesion-only revascularization in patients undergoing primary percutaneous coronary intervention for STEMI and multivessel disease: the CvLPRIT trial. J Am Coll Cardiol 2015; 65: 963-72. [Crossref]

7. Smits P, Omerovic E, Abdel-Wahab M, Boxma-de Klerk B, Lunde K, Neumann FJ, et al. Two year results from the compare-acute trial. J Am Coll Cardiol 2018; 71 (11 Supplement): A1158. [Crossref]

8. Mehta SR, Wood DA, Storey RF, Mehran R, Bainey KR, Nguyen H, et al.; COMPLETE Trial Steering Committee and Investigators. Complete Revascularization with Multivessel PCI for Myocardial Infarction. N Engl J Med 2019; 381: 1411-21. [Crossref]

9. Serruys PW, Morice MC, Kappetein AP, Colombo A, Holmes DR, Mack MJ, et al.; SYNTAX Investigators. Percutaneous coronary intervention versus coronary-artery bypass grafting for severe coronary artery disease. N Engl J Med 2009; 360: 961-72. [Crossref]

10. Tarantini G, D'Amico G, Brener SJ, Tellaroli P, Basile M, Schiavo A, et al. Survival After Varying Revascularization Strategies in Patients With ST-Segment Elevation Myocardial Infarction and Multivessel Coronary Artery Disease: A Pairwise and Network Meta-Analysis. JACC Cardiovasc Interv 2016; 9: 1765-76. [Crossref]

11. Ochala A, Smolka GA, Wojakowski W, Dudek D, Dziewierz A, Krolikowski Z, et al. The function of the left ventricle after complete multivessel one-stage percutaneous coronary intervention in patients with acute myocardial infarction. J Invasive Cardiol 2004; 16: 699-702.

12. Hachet O, Guenancia C, Stamboul K, Daubail B, Richard C, Béjot $Y$, et al. Frequency and predictors of stroke after acute myocardial infarction: specific aspects of in-hospital and postdischarge events. Stroke 2014; 45: 3514-20. [Crossref]

13. Scarsini R, Terentes-Printzios D, De Maria GL, Ribichini F, Banning A. Why, When and How Should Clinicians Use Physiology in Patients with Acute Coronary Syndromes? Interv Cardiol 2020; 15: e05. [Crossref]

14. Wood DA, Poulter RS, Boone R, Lim I, Bogale N, Starovoytov A, et al. Stability Of Non-Culprit Vessel Fractional Flow Reserve In Patients With ST-Segment Elevation Myocardial Infarction. JACC online 2013 Oct. Available from: URL: https://www.jacc.org/doi/ pdf/10.1016/j.jacc.2013.08.1376?download=true [Crossref]

15. Ntalianis A, Sels JW, Davidavicius G, Tanaka N, Muller O, Trana C, et al. Fractional flow reserve for the assessment of nonculprit coronary artery stenoses in patients with acute myocardial infarction. JACC Cardiovasc Interv 2010; 3: 1274-81. [Crossref]

16. Koh JS, Samady H. Robustness of Fractional Flow Reserve for Lesion Assessment in Non-Infarct-Related Arteries of Patients With Myocardial Infarction. J Am Heart Assoc 2019; 8: e012456. [Crossref]

17. Choi KH, Lee JM, Kim HK, Kim J, Park J, Hwang D, et al. Fractional Flow Reserve and Instantaneous Wave-Free Ratio for Nonculprit Stenosis in Patients With Acute Myocardial Infarction. JACC Cardiovasc Interv 2018; 11: 1848-58. [Crossref] 
Appendix A. Criteria for inclusion and exclusion

\section{Inclusion criteria:}

1. Men and women after successful percutaneous coronary intervention (PCI) (preferably using a drug-eluting stent) to the culprit lesion for ST-elevation myocardial infarction (STEMI). PCI for STEMI should be primary PCI in the first 12 hours after symptom onset.

2. Multi-vessel disease defined as at least 1 additional non-infarct related coronary artery lesion that is at least $2 \mathrm{~mm}$ in diameter that has not been stented as part of the primary $\mathrm{PCl}$ and is amenable to successful treatment with $\mathrm{PCl}$ and has:

0 At least $75 \%$ diameter stenosis (visual estimation) or

0 At least $50 \%$ diameter stenosis (visual estimation) with fractional flow reserve $\leq 0.80$

3. Age between 18 and 90 years

4. The interventional cardiologist had to regard $\mathrm{PCl}$ as a valid option to treat all significant stenoses (infarct related artery (IRA) and non-IRA related).

5. Written informed consent should be obtained from all the patients after the primary PCI.

\section{Exclusion criteria:}

1. Rescue $\mathrm{PCl}$ for failed fibrinolysis or a combination strategy where $\mathrm{PCl}$ is performed routinely $3-12$ hours after fibrinolysis.

2. Cardiogenic shock

3. Cardiac arrest

4. Planned surgical revascularization (Syntax and STS score were calculated, and the best treatment [coronary artery bypass graft (CABG) or PCI] was presented to the patient. If the patient opted for $\mathrm{CABG}$, they would not be eligible for inclusion and randomization).

5. Non-cardiovascular known co-morbidity reducing life expectancy to $<2$ years.

6. Any factor precluding one-year follow-up.

7. Prior CABG surgery.

8. A different operator from the previously designated.

9. Unable to provide consent for any other reason.

10. Impaired renal function (GFR $<60 \mathrm{~mL} / \mathrm{min} / 1.73 \mathrm{~m}^{2}$ ). 